\title{
Load rating assessment of masonry arch bridges
}

\author{
P. Řeřicha* \\ Czech Technical University in Prague, Faculty of Civil Engineering, Czech Republic \\ *Corresponding author: rer@cml.fsv.cvut.cz
}

\begin{abstract}
Simple formulas are developed for the mass scale load rating assessment of masonry arch bridges in terms of four geometrical parameters of the bridge. The ultimate load limit state and the repeated load limit state constitute the theoretical background. The formulas are constructed as the minimum squares best suited to a set of linear and nonlinear finite element solutions of a representative selection of the country's bridge stock. The formulas are quadratic in the arch span and linear in the arch rise, arch thickness and fill depth. The method can be used for other countries' masonry arch bridge stock; the data processing programme is portable. The structure of the formulas and the country's bridge stock representation can be adapted to local conditions. Nevertheless, new representations of bridge instances require rather demanding non-linear finite element solutions up until total collapse. This is the most laborious part of the formula's development.
\end{abstract}

KEY WORDS: Arch bridges, masonry, load rating, limit states, finite element method, semiempiric formulas

\section{INTRODUCTION}

Load rating of masonry arch bridges is important for road maintenance and management. Its economic impact is considerable, owing to the large numbers of these structures in all developed countries. For the same reason, the assessment methods should be simple and applicable on a mass scale. Also, inevitable uncertainties in material properties make the use of sophisticated analytical methods disputable. The Czech Ministry of Transport therefore funded the development of a manual for the load rating of the country's masonry arch bridges, referred to as the Guide in the paper. The Guide is designed for local bridge engineers with standard civil engineering education and practice. The simplicity requirement admits only semi-empirical formulas. Pippard's formula, (Pippard 1938), see also Heyman (1982) became the basis of the MEXE load rating method, devised in the 1950s in a British military experimental establishment, which in turn has been adapted to several guides and is widely used today, (Highway Agency 1997, UIC 1995 and Min. of Transport CR 2000). Failure criteria play an important part in the development of semi-empirical formulas. The middle half rule is accommodated in Pippard's formula, whereas a relative thrust line eccentricity of 0.35 is allowed in Czech assessment tables, (Min. of Interior CR, 1989). A common deficiency of approximate methods and formulas is that the interaction of the barrel, fill, abutments and roadway is not properly accounted for. As for the analytical methods, the greatest effort has been directed toward the accurate simulation of ultimate limit load states (ULS) and bridge collapse, see (Owen 1998, Fanning, Boothby, Roberts 2001 and Fanning, Boothby 2003) among others. The load rating, i.e., the load capacity for service conditions, is then derived as a fraction of the ultimate limit load. Probably the most advanced and cited British design manual, Highway Agency (2001), recommends a safety 
factor of $\gamma=3.4$, whereas, when the standard structural reliability factors of Eurocodes are combined, the reduction factor is 2.17. The latter value applies to the ultimate load based on characteristic values of material strengths and no dynamic factor is included. The nature of the typical collapse, namely, the stability loss when four virtual hinges arise, see (Highway Agency 2001), does not admit the standard partial safety factor approach. A consistent probabilistic reliability theory fails, too, since necessary stochastic data is missing. The reduction of the ultimate load to the load rating for the service load is speculative and uncertain. Two concepts are utilized in the Guide development in order to reduce uncertainty.

First, the ULS is not the only limit state from which the load rating is derived; a repeated load limit state (RLLS) is introduced. Second, the safety factor in the ULS is calibrated by comparison to other load rating methods. The Guide features these basic concepts:

1. The load rating method and structure models do not account for abutment, piers and foundation compliance and failure. These structural parts require individual treatment that can hardly be condensed in a common guide.

2. Two limit states, the ULS and the repeated load limit state (RLLS), defined below, determine the load rating.

3. Simple semi-empirical formulas are derived as minimum squares best suited to a set of numerical finite element linear and non-linear solutions of a representative set of bridge instances termed the representation.

4. Besides the direct semi-empirical formula for the load rating, criteria are provided for an elaborate assessment by linear numerical analysis. Their limit values follow from semi-empirical formulas as well.

5. The two methods developed for the load rating, the direct semi-empirical formula and numerical analysis, are hierarchical as to their precision, work load and use.

\section{REPEATED LOAD LIMIT STATE}

In practice, most bridges are put out of service when excessive deterioration of masonry occurs, particularly deterioration of the joints. This can be considered another limit state which belongs to the serviceability limit states in the CEN ENV 1991-1 1994 nomenclature. The associated limit load is a repetitive vehicle passage that does not induce cumulative persistent damage in the bridge structure. Experience shows that moderate cracks in the barrel can stabilize. The existence of cracks alone therefore is not a suitable criterion of the limit state. The relative crack depth $c_{0}$ (with respect to the arch thickness) is adopted herein for the quantity to compare in this limit state. It is assumed that a crack depth limit $c_{0, \text { lim }}$ exists for 'harmless' cracks, an analogy to the endurance limit in fatigue. Unfortunately, sufficient experimental evidence on $c_{0, \lim }$ will not be available in the foreseeable future. Despite this, the repeated load limit state (RLLS), is adopted for the second limit state in the project. The limit crack depth is determined by calibration and expert agreement, which is common practice in design standard criteria selection. Note that non-linear structural analysis is indispensable for the RLLS solution in spite of the fact that it is a serviceability limit state.

Recently, Melbourne et all (2007) proposed a similar limit state, the permissible limit state (PLS). The purpose of the PLS is similar. It is also unique for masonry arch bridges and should be additionally checked with the standard ultimate load limit state. Its definition is "the limit at which there is a loss of structural integrity that will measurably affect the ability of the bridge to carry its working loads for the expected life of the bridge". Criteria of the PLS depend on the failure mode and are not definitely specified in the paper. 
The authors present an example in which the criteria are the endurance limit compressive stress in bending and the longitudinal shear stress between rings in rings separation. The former is one half of the compressive strength with vague justification, the latter was determined from a series of laboratory tests $(0.1 \mathrm{Mpa})$. It is difficult to imagine how the endurance limit shear stress could be determined for an existing bridge. Nevertheless, the paper indicates the need for a specific limit state for masonry arch bridges.

\section{PARAMETER RANGES AND THE BRIDGE POPULATION REPRESENTATION}

The ULS and RLLS are difficult to account for in simple assessment formulas. The approach adopted herein is based on numerical linear and non-linear solutions of the representation and their best fit approximation by the target semi-empiric formulas. Prospective users of the formulas need not know about the subtleties of the non-linear numerical solutions. Semiempiric formulas must have applicability ranges specified on their parameters, which should cover the country's bridge population as much as possible. A survey of masonry arch bridge stock in the country has been conducted with a statistical assessment of their parameters. On this basis, a decision has been made on the ranges of the parameters to be covered by the target formula and by the representation. The outcome ranges of the geometrical parameters

$$
2<l<8[\mathrm{~m}], \quad 0.15<h / l<0.5,0.07<d / l<0.20, \quad 0.08<s / l<0.45
$$

cover $95 \%$ of the country's stock. Parameters are shown in Fig.1.

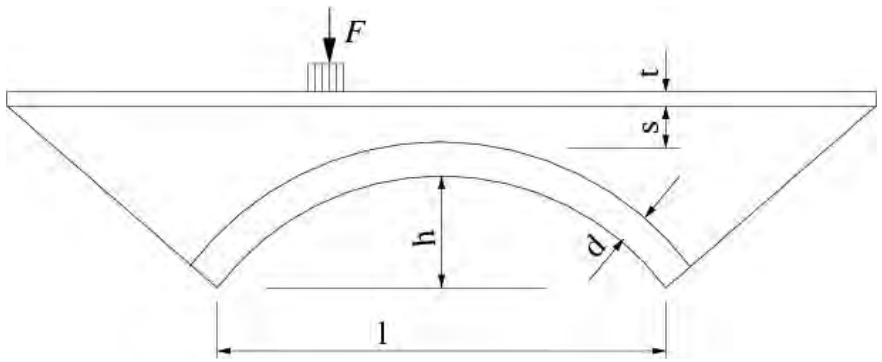

Figure 1: Schematic longitudinal section, method parameters

The parameter ranges govern the selection of bridge instances to be included in the representation and analysed. The survey did not identify any multi-ring arches so ring separation is not considered in the representation analyses. The results presented herein are based on a representation including 37 instances. They do not quite regularly cover the four-dimensional space of parameters (three values per parameter mean $3^{4}=81$ instances). Convenience of mesh generations and similar aspects play a part in the instances selection. The representation is displayed in Fig. 4. The format of the instance legends is $l-h-d-s$. Note that absolute lengths are used in the legends.

\section{FINITE ELEMENT LINEAR AND NON-LINEAR SOLUTIONS OF THE REPRESENTATION, BRIDGE STRUCTURE AND LOAD MODELS}

A simple target formula demands a simple structure model. It is assumed that load carrying capacity depends mainly on the longitudinal bridge section. A plain strain $2 D$ structure model is therefore sufficient to disclose the principal dependencies. This does not preclude 3D 
analyses of individual structures as options in the numerical method (see section 6). Properties and parameters associated with the cross-sections of the bridges are included 'ex post' in approximate corrections if necessary. This approach is rather common in theoretical and experimental bridge load capacity assessments and load rating methods since it saves full $3 \mathrm{D}$ analyses. A schematic longitudinal section of the bridge in Fig. 1 exposes the bridge parameters to be represented in the target formula, barrel span $l$, barrel rise $h$, barrel thickness $d$, and fill thickness $s$ above the barrel crown. These parameters appear in the mathematical formulation of the best fit problem specified in section 5. The inclination of the fill wedges was approximately the same for all representation instances. The wedges provide approximately correct boundary conditions for the fill volume above the arch that takes part in the live load transition. They are fixed at the external (lower) boundaries and so are the ends of the arch. An average thickness $t=0.15 \mathrm{~m}$ is assumed for the roadway.

Material models and constants are the same in the whole representation, see Table 1, and do not appear as variables in the formulas. This seems to be too radical an assumption, since the bridge load rating does not depend on the properties of the materials. Nevertheless, it has been used several times already, by Heyman (1982) and Fanning \& Boothby (2003). The failure criteria of four bridges assessed in (Boothby, 2001) deliver the same results regardless of material strengths. These references testify that the assumption is acceptable. However, the main reason for its adoption is that material constants are not available for the formula's users. In routine applications, an engineer has only the results of a visual inspection with no quantitative data on material properties. A proper probe by (mostly) nondestructive methods would often cost more than the bridge itself.

For simplicity and easy comparison with foreign solutions, no tension material is assumed in the arch. The barrel in plain strain conditions can be modelled by $2 \mathrm{D}$ elements or by shell elements which reduce it to a $1 \mathrm{D}$ continuum.

Three FEM non-linear packages have been used. Most computations have been done by Adina R\&D, (2004), several results checked with Atena by Cervenka Consulting, (2007) and with an in-house code by Rericha, (2000), using the mesh generator by Rypl, (2004). All three packages feature similar material models and finite element types. A difference worth mentioning is that the third package supports layered shell elements with a suitable material model whereas in the first two, isoparametric 2D elements are used for the arch. The distorted mesh is shown in Fig. 2 prior to collapse for an instance of the representation. The cracked parts of the joints are indicated and plastic strain contours in the fill are displayed.

Important restrictions are made on the live load variability. With regard to the barrel span bounds eq. (1), the moving vehicle is reduced to a single axle (the rear axle of a truck) in a lane. Further, a single position of the axle in the span direction is considered at $1 / 4$ of the barrel span. If full variability of the live load were considered according to most standards, the determination of limit states would become unmanageable. Reduction to 2D plain strain conditions entails the replacement of the actual axle pressure pattern by a uniform pressure strip in the lateral direction. The pressure strip is indicated by the double force in Fig. 2. Most papers cited in the introduction section adopt a similar live load proxy. 


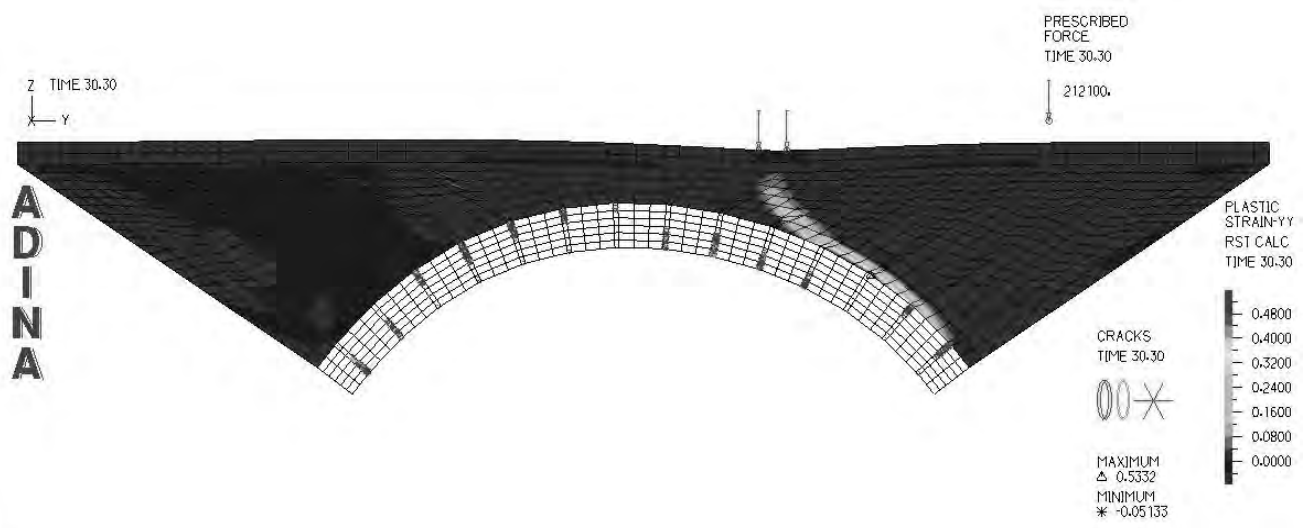

Figure 2: Finite element mesh, plastic zone in the fill and cracks in the arch joints at the ultimate load level for an instance bridge

Tab. 1: Material models and constants, units $\mathrm{m}, \mathrm{t}, \mathrm{s}, \mathrm{kPa}$

\begin{tabular}{|c|c|c|c|c|c|c|c|c|}
\hline & $\begin{array}{c}\text { Young } \\
\text { modulus }\end{array}$ & $\begin{array}{c}\text { Poisson } \\
\text { ratio }\end{array}$ & density & $\begin{array}{c}\text { compr. } \\
\text { strength }\end{array}$ & $\begin{array}{c}\text { tension } \\
\text { strength }\end{array}$ & cohesion & $\begin{array}{c}\text { friction } \\
\text { angle }\end{array}$ & $\begin{array}{c}\text { yield } \\
\text { stress }\end{array}$ \\
\hline arch & $3.0+06$ & 0.15 & 2.0 & -3000. & 10. & & & \\
\hline $\begin{array}{c}\text { fill, Mohr } \\
\text { Coulomb }\end{array}$ & $4.0+4$ & 0.30 & 1.7 & & & 10. & 0.50 & \\
\hline $\begin{array}{c}\text { roadway, } \\
\text { Mises }\end{array}$ & $5.5+06$ & 0.20 & 2.0 & & & & & 6000. \\
\hline
\end{tabular}

Table functions ej(f) and $\mathrm{c} 0, \mathrm{j}(\mathrm{f})$ are recorded from the linear and non-linear analyses of each instance $\mathrm{j}$ where $\mathrm{f}$ denotes the load factor for the live load, ej(f) is the maximum relative thrust eccentricity in the linear solution of the instance $\mathrm{j}$ and $\mathrm{c} 0, \mathrm{j}(\mathrm{f})$ is the maximum relative crack depth in the non-linear solution. Maximum within the arch is meant here and relative refers to the arch thickness. Eliminating $\mathrm{f}$ yields functions ej(c0) which are further processed together with ultimate load factors fULS,j.

\section{RAW DATA PROCESSING AND PARAMETER CALIBRATION}

The criterion of the RLLS, $c_{0}<c_{0, \text { lim }}$, is not acceptable for mass use since a non-linear bridge structure analysis is necessary on the part of the Guide user with every instance. In order to avoid it, a correlation is assumed between the crack depth in a non-linear analysis and the relative thrust force eccentricity in a linear analysis in the same bridge instance (at the same load level). The correlation is approximated by a quadratic function of $c_{0}$ whose coefficients are functions of the bridge parameters, specified below.

The RLLS criterion converts to $e<e_{\text {lim }}$ in the wake of the correlation.

Table functions $e_{j}\left(c_{0}\right)$ and ultimate limit loads $f_{U L S, j}$ of all representation bridge instances are processed by a single purpose programme to obtain their least square approximations

$e\left(c_{0}, l, h, d, s\right)$ and $f_{U L S}(l, h, d, s)$. Actually, the approximations are linear in $h, d, s$ and quadratic in $l$. For given bridge structure parameters and limit crack depth $c_{0, \text { lim }}$, the ultimate limit load and limit relative eccentricity follow from these two approximations. Using the inverse functions to $e_{j}(f)$, the RLLS limit loads $f_{R L L S, j}$ are computed from the limit relative eccentricities. The final load ratings of the representation are $f_{C A P, j}=\min \left(f_{R L L S, j,}, \gamma f_{U L S, j}\right)$ where $\gamma$ is the safety factor with respect to the ULS. These are once again fitted by the least 
square approximation $f_{C A P}(l, h, d, s)$. This function and the limit relative eccentricity $e_{l i m}=e\left(c_{0, l i m}, l, h, d, s\right)$ are the results of raw data processing additionally to $f_{U L S}(l, h, d, s)$.

Two values are subject to calibration, the admissible relative crack depth in the RLLS, $c_{0, \text { lim }}$, and the safety factor $\gamma$ of the ULS. Calibration is supported by a graphic display of the intermediate results. An example is shown in Fig. 4, where the values $c_{0, \text { lim }}=0.4$ and $\gamma=3.4$ are used and the semi-empiric formula results are compared to the MEXE method (Highways Agency, 1997) and the current Czech standard (Min. of Interior of the CR, 1989). On the vertical axis there are forces per $1 \mathrm{~m}$ wide strip of the arch. Comparable MEXE values are obtained by applying the span-rise and profile factors to the PAL values and dividing them by the lane width $3 \mathrm{~m}$. Other factors of the MEXE method account for the material and condition and are not used to obtain comparable values.

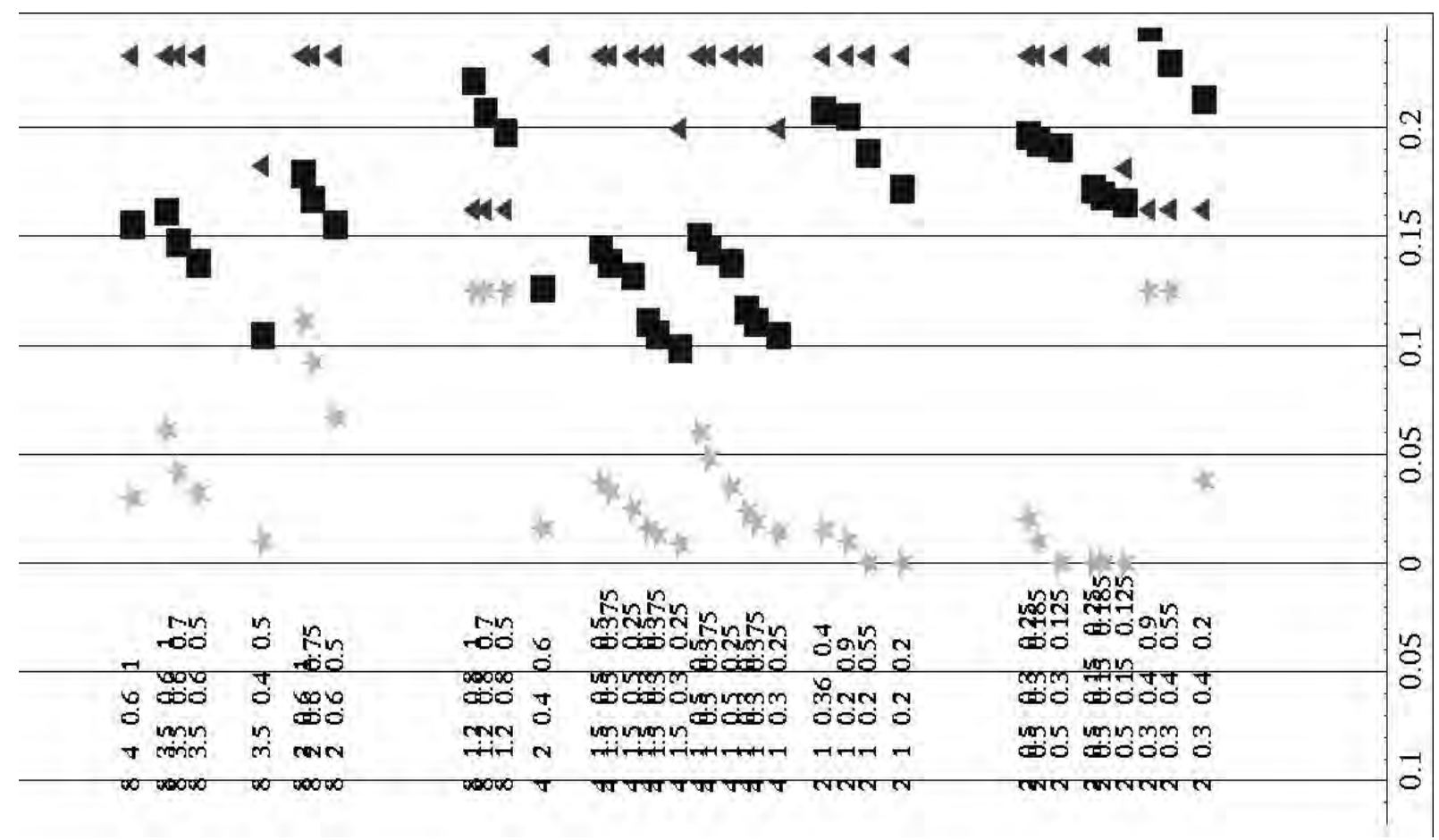

Figure 3: Load ratings of the representation [MN/m], vertically, ULS safety margin $\gamma=3.4$, limit relative crack depth $c_{0, \text { lim }}=0.4$. Blue squares - semi-empiric formula, red triangles - MEXE method, green asterisks - Czech standard. Instances of the representation are allocated on the horizontal axis by increasing parameters $l, h, d$ and $s$ (barrel span, rise and thickness and fill depth).

Vertical alignment of most MEXE values corresponds to the upper bound $70 \mathrm{t}$ of the PAL and testifies that the MEXE method is not suitable for the country's arch bridge stock. The Czech standard values on the other hand exhibit inadequate scatter and conservatism. The semi-empiric formula offers substantial improvement. The calibration process remains open in that the representation can be extended. The current values of $c_{0, \text { lim }}$ and $\gamma$ may also change in the future. In particular, $c_{0, \text { lim }}$ should be bolstered by experimental evidence. Tests on eccentric compression of masonry specimens with cyclic reversed load are being prepared which should confirm the existence of the repeated load limit state and the suitability of its criterion. Processing of the linear and non-linear representation analyses results is programmed in Mathematica (Wolfram Research Ltd, 2006) including graphic output for easy calibration. The programme is available free upon request to the author. Most numerical representation solutions, however, can hardly be ported to other country conditions. 


\section{THE GUIDE APPLICATION}

The user checks first whether the bridge parameters and condition comply with Guide applicability conditions and parameter ranges. For routine use, the load rating formula $f_{C A P}$ $(l, h, d, s)$ is explicitly given. The load rating is readily obtained by substitution of the actual bridge parameters. For later reference, this is termed the direct formula method.

There are instances of particular importance when a more precise evaluation is desirable. The Guide offers an option for those cases when $f_{U L S}(l, h, d, s)$ is greater than $f_{C A P}(l, h, d, s)$, that is, for the cases when the RLLS is decisive in the load rating assessment. A more elaborate structural model can produce a less conservative rating than the direct formula in these cases. The Guide provides a formula for the limit relative thrust eccentricity $e_{\text {lim }}=e\left(c_{0, l i m}, l, h, d, s\right)$. The user performs a standard linear analysis of the bridge structure. The analysis should be performed with an adequate structure model accounting for the interaction of the barrel, fill, roadway and spandrels, true 3D distribution of the load and structural resistance. A finite element method will almost exclusively be used. The analysis is quasi-static, considering the superposition of the dead load and a proportionally growing live load according to the respective road load standard. The maximum thrust force eccentricity within the barrel grows with the growing live load level. When the limit relative thrust eccentricity $e_{\text {lim }}$ is reached, the RLLS limit load $f_{R L L S}$ is achieved. It will be greater than the load rating by the direct formula in most cases. The final load rating is $\min \left(f_{R L L S}, \gamma \quad f_{U L S}\right)$. This is termed the numerical solution method. Its cost is incomparable to the direct formula method and its application will be rather exceptional. It may turn out to be acceptable with skew bridges, integral spandrel action and similar spatial phenomena.

\section{CONCLUSIONS}

A load rating method is developed for masonry arch bridges, suitable for mass scale application. Two hierarchical procedures are proposed, linear elastic numerical analysis and semi-empiric formula. Both are based on two limit states, the ultimate load state and the repeated load limit state. The actual calibrated values of the limit crack depth and ultimate load safety factor yield load ratings better than the current Czech standard or the MEXE. The draft Guide with user instructions is entering the formal commissioning process to become official.

\section{ACKNOWLEDGEMENT}

The support through project 1F55A/005/120 of the Ministry of Transport of the Czech Republic is acknowledged.

\section{REFERENCES}

Adina R\&D, 2004: ADINA Automatic Dynamic Incremental Nonlinear Analysis, 8.1.3 edition, Watertown, Mass., USA.

Boothby T.E., 2001: Load Rating of Masonry Arch Bridges. Proc. Inst. Civil Engineers, Bridge Engineering, 6, No 2, 79-86.

CEN ENV 1991-1, 1994: Basis of Design and Actions on Structures Part 1: Basis of Design. Eurocode 1. 
Cervenka Consulting, 2007: ATENA Atena program documentation, www.cervenka.cz.

Fanning,P.J., Boothby, T.E, Roberts, B.J., 2001: Longitudinal and Transverse Effects in Masonry Arch Assessment. Construction and Building Materials, 15,51-60.

Fanning,P.J., Boothby, T.E., 2003: Experimentally-based assessment of masonry arch bridges. Proceedings of the Institution of Civil Engineers, Bridge Engineering, 156, No. 3,109-116.

Pippard, A.J.S., Ashby R.J.: An experimental study of a voussoir arch. Proc. Inst. Civil Engrs, $1938,10,383$.

Heyman, J.: The masonry arch. Ellis Harwood, Chichester, 1982.

Highways Agency, 1997: The assessment of highway bridges and structures, amendment no 2, chapter 3, Assessment masonry arch bridges by the modified MEXE method. Design manual for roads and bridges, vol 3,section 4, part 4. Her majesty's Stationery Office, London, 1997, BA 16/97.

Melbourne, C., Wang, J., Tomor, A.K., 2007: A new masonry arch bridge assessment strategy (SMART). Proc. ICE, Bridge Engineering, Vol.160, No.2, p. 81-87.

Min. of Interior of the CR, 1989: Load rating tables. Appendix 1 Guide to load rating arch bridges on motorways, roads and local roads (in Czech).

Min. of Tranport of the CR, 2000: Recommendations for design of the new and rating of the existing concrete road bridges (in Czech). TP144.

Owen, D.J.R. et all., 1998: Finite/discrete element method for assessment and repair of masonry structures. In A. Sinopoli, editor, Arch bridges, Proc. 2nd International Arch Bridge Conference. Balkema.

Rericha, P., 2000: Static and dynamic limit loads of reinforced concrete structures, CTU Reports vol.4., Czech Technical University in Prague.

Rypl, D., 2004: T3D mesh generator. Czech Technical University in Prague, Faculty of Civil Engineering, http://mech.fsv.cvut.cz/dr/t3d.html.

UIC, 1995: Recommendations for the assessment of the load carrying capacity of existing masonry and mass-concrete bridges, UIC Code 778-3r. Int. Union of Railways, 16 Rue Jean Rey - 75015 Paris.

Wolfram Research Ltd, 2006: Mathematica 5.2. Wolfram Research Ltd., 10 Blenheim Office Park, Lower Road, Long Hanborough, Oxfordshire OX29 8RY, U.K., 2006, www.wolfram.com. 